\title{
Effects of Oral $\alpha$-Lipoic Acid on Blood Pressure and Inflammatory Factors in Overweight or Obese Subjects: A Randomized, Double-Blind, Placebo- Controlled Trial
}

\author{
Nong $\mathrm{Li}^{1}$, Meiyu Zhang ${ }^{2 *}$, Xiaojuan $\mathrm{Hu}^{2}$, Fugang Wang ${ }^{1}$, Cheng $\mathrm{Li}^{3}$, Kehong Sun${ }^{2}$, Aixia Xie ${ }^{1}$ and \\ Xuefang Huang ${ }^{1}$ \\ ${ }^{1}$ Department of Endocrine and Metabolic Diseases, People's Hospital of Kelamay city, Kelamay city, Xinjiang, China \\ ${ }^{2}$ Medical Center of the People's Hospital of Karamay, Kelamay city, Xinjiang, China \\ ${ }^{3}$ Information Center of the First Affiliated Hospital of Xinjiang Medical University, Urumqi City, Xinjiang, China \\ *Corresponding author: Meiyu Zhang, Department of Endocrine and Metabolic Diseases, Medical Center of the People's \\ Hospital of Karamay, Karamay city 834000, China
}

ARTICLE INFO

Received: May 16, 2020

Published: 蔧 June 02, 2020

Citation: Nong Li, Meiyu Zhang, Xiaojuan $\mathrm{Hu}$, Fugang Wang, Cheng Li, et al., Effects of Oral $\alpha$-Lipoic Acid on Blood Pressure and Inflammatory Factors in Overweight or Obese Subjects: A Randomized, Double-Blind, Placebo-Controlled Trial. Biomed J Sci \& Tech Res 28(1)-2020. BJSTR. MS.ID.004591.

Keywords: $\alpha$-Lipoic Acid; Overweight/ Obesity; Blood Pressure; Inflammatory Factors

Abbrevations: CVD: Cardiovascular Disease; CHD: Coronary Heart Disease; CRP: C-Reactive Protein; ALA: $\alpha$-Lipoic Acid; CAD: Coronary Artery Disease; FPG: Fasting Plasma Glucose; GSH: Glutathione

\section{Summary}

Objective: To evaluate the effects of the antioxidant $\alpha$-lipoic acid (ALA) after oral administration on blood pressure and inflammatory factors in overweight or obese subjects.

Methods: This was a randomized, double-blind, placebo-controlled crossover clinical trial. A total of 103 subjects of Chinese Han ethnicity were randomly assigned into ALA $(n=52)$ or placebo groups $(n=51)$. Subjects in the ALA group were treated with oral ALA $1200 \mathrm{mg} /$ day for 8 weeks, followed by a 4-week washout period, then received placebo for another 8 weeks. Subjects in the placebo group were administered with placebo for 8 weeks, followed by a 4 -week washout period and another 8 -week treatment with ALA (1200mg/day).

Result: After 8 weeks' intervention, there were no significant differences in terms of the reduction in brachial-ankle pulse wave velocity $(-26.30 \pm 117.23 \mathrm{~cm} / \mathrm{s}$ vs. $4.23 \pm 120.78 \mathrm{~cm} / \mathrm{s}, \mathrm{p}=0.111)$,interleukin $-6(-22.51 \pm 37.11 \mathrm{pg} / \mathrm{ml}$ vs. $-30.39 \pm 53.47 \mathrm{pg} /$ $\mathrm{ml}, \mathrm{p}=0.552)$, C-reactive protein $(-0.85 \pm 5.92 \mu \mathrm{g} / \mathrm{ml}$ vs. $-0.83 \pm 5.96 \mu \mathrm{g} / \mathrm{ml}, \mathrm{p}=0.884)$, or diastolic blood pressure $(5.44 \pm 8.96 \mathrm{mmHg}$ vs. $7.19 \pm 10.08 \mathrm{mmHg}, \mathrm{p}=0.121)$ compared with the placebo group. However, systolic blood pressure (SBP) $(-1.25 \pm 8.59 \mathrm{mmHg}$ vs. $2.29 \pm 9.12 \mathrm{mmHg}, \mathrm{p}=0.029$ ) in the ALA group were significantly lower than the placebo group. After adjusting for differences in sex, age, treatment sequence and period, the results of mixed-effects models showed that the differences in SBP between the two groups were significant before and after the intervention.

Conclusion: Treatment with ALA was associated with no significant reduction in the markers of inflammation in overweight or obese subjects. However, ALA treatment may lead to decreased SBP.

\section{Introduction}

Recently, the prevalence of obesity has rapidly increased around the world, which is considered the most important cause for the dramatic increase in the incidence of cardiovascular disease (CVD) and type 2 diabetes (T2D). Obesity is a significant risk factor leading to CVD and T2D [1-3]. Although the influence of obesity as an independent risk factor for CVD is still controversial and requires further research, individuals with obesity are often complicated by metabolic syndrome (MetS), such as hypertension, hyperlipemia, 
and impaired glucose regulation, which can increase the risk of CVD onset and lead to significantly increased cardiovascular morbidity and mortality $[4,5]$.

It has previously been found that there is a significant positive correlation between biomarkers of oxidative stress (OS) and body mass index (BMI) [6]. OS caused by obesity has been shown to have a crucial role in the development of CVD, such as atherosclerosis, coronary heart disease (CHD), and hypertension, and is regarded as the pathogenic mechanism underlying CVD and diabetes $[7,8]$. Several studies have shown that obesity is independently associated with OS $[6,9,10]$. Elevated levels of OS in obese subjects can increase vascular endothelial permeability, destroy vascular structure and endothelial function, and lead to increased wall thickness, lumen narrowing, and increased vasoconstriction, thereby resulting in the development of CVD [11,12]. In addition, obesity also represents a state of chronic low-level inflammation $[13,14]$. The inflammatory responses in obese subjects, which also result from OS, increase with the obesity [15], and OS could activate the pathway related to inflammatory responses. It has been shown that these inflammatory responses are involved in the development of atherosclerosis $[16,17]$, and the incidence of CVD increases with the elevation of the inflammatory marker C-reactive protein (CRP) [18]. Thus, OS and inflammatory responses in obese subjects may be common mechanisms involved in the pathogenesis of obesityinduced CVD.

Collectively, these findings suggest that the multiple mechanisms involved in CVD and diabetes are associated with OS; and $O S$ is a cause of endothelial cell injury and dysfunction, as well as CVD complications such as atherosclerosis. Therefore, antioxidant supplementation that is effective in reducing OS might be a strategy for the prevention and treatment of CVD. $\alpha$-lipoic acid (ALA) is a powerful antioxidant with various antioxidant functions. ALA can improve endothelial function and reduce inflammatory markers in patients with MetS [19]. In addition, ALA has been shown to be effective in the treatment of diabetic polyneuropathy $[20,21]$. However, the role of ALA on CVD risk factors such as brachial-ankle pulse wave (baPWV), inflammatory factors, and blood pressure has not been fully elucidated. We therefore evaluate the effects of oral ALA on blood pressure and inflammatory factors in overweight or obese subjects, and the mechanisms of ALA were also explored.

\section{Subjects and Methods}

\section{Subjects}

From May to October 2009, subjects of Chinese Han ethnicity who were underwent a physical examination at the Medical Center of the People's Hospital of Karamay, Xinjiang, China were enrolled in the study. The inclusion criteria were: (1) subjects of Han ethnicity, aged 20-60 years, with a BMI $\geq 25 \mathrm{~kg} / \mathrm{m} 2$; (2) subjects who were not taking any antioxidant supplements prior to inclusion; and (3) subjects who had at least one type of the following diseases: (i) borderline hypertension $(130 \mathrm{mmHg} \leq$ systolic blood pressure (SBP) $<140 \mathrm{mmHg}$ and/or $85 \mathrm{mmHg} \leq$ diastolic blood pressure (DBP) < 90mmHg); (ii) abnormal blood lipid levels (total cholesterol (TC) $\geq 5.2 \mathrm{mmol} / \mathrm{l}$ or high-density lipoprotein cholesterol (HDL-C) $<1.04 \mathrm{mmol} / \mathrm{l})$; (iii) impaired fasting glucose $(6.1 \mathrm{mmol} / \mathrm{l}$ blood glucose (FBG) $<7.0 \mathrm{mmol} / \mathrm{l}$ ).

Subjects who met the following criteria were excluded from the study:

a) Pregnant, lactating, or women of child-bearing age who were not using any method of contraception.

b) Subjects with hypertension, diabetes, coronary artery disease (CAD) cancer, or liver disease.

c) Subjects who could not understand the study or who refused to participate, and those with a psychiatric history were also excluded. A total of 347 subjects were preliminary screened, and 166 subjects who fulfilled the inclusion and exclusion criteria were enrolled. Written informed consent was obtained from 103 subjects, including 63 males and 40 females.

\section{Randomization}

This study was a randomized, double-blind, placebo-controlled crossover clinical trial. After consent was obtained, subjects were sequentially entered into the study using a blocked randomization schedule, and randomly assigned to two groups using Excel in a double-blind fashion, with a balanced sex ratio. The randomization sequence was generated by the principal investigators, and two investigators were in charge of registration and assignment.

\section{Intervention Protocols}

ALA or placebo tablets (both offered by the Medical Research Institute, San Francisco, CA, USA) were administered orally at a twice-daily dose of $1200 \mathrm{mg}$ (30 minutes before breakfast or dinner) for an intervention period of 8 weeks. Medication compliance was assessed via telephone interviews during this period. Subjects in the ALA group were treated with oral ALA $1200 \mathrm{mg}$ /day for 8 weeks, followed by a 4 -week washout period, then received placebo for another 8 weeks. Subjects in the placebo group were administered placebo for 8 weeks, followed by a 4 -week washout period and a further 8-week treatment with ALA (1200mg/day). Therefore, all subjects received ALA for 8 weeks (Figure 1). The study protocol was carried out according to the Declaration of Helsinki and Good Clinical Practice requirements and approved by the ethics committee of the People's Hospital of Kalamay, Xinjiang. 


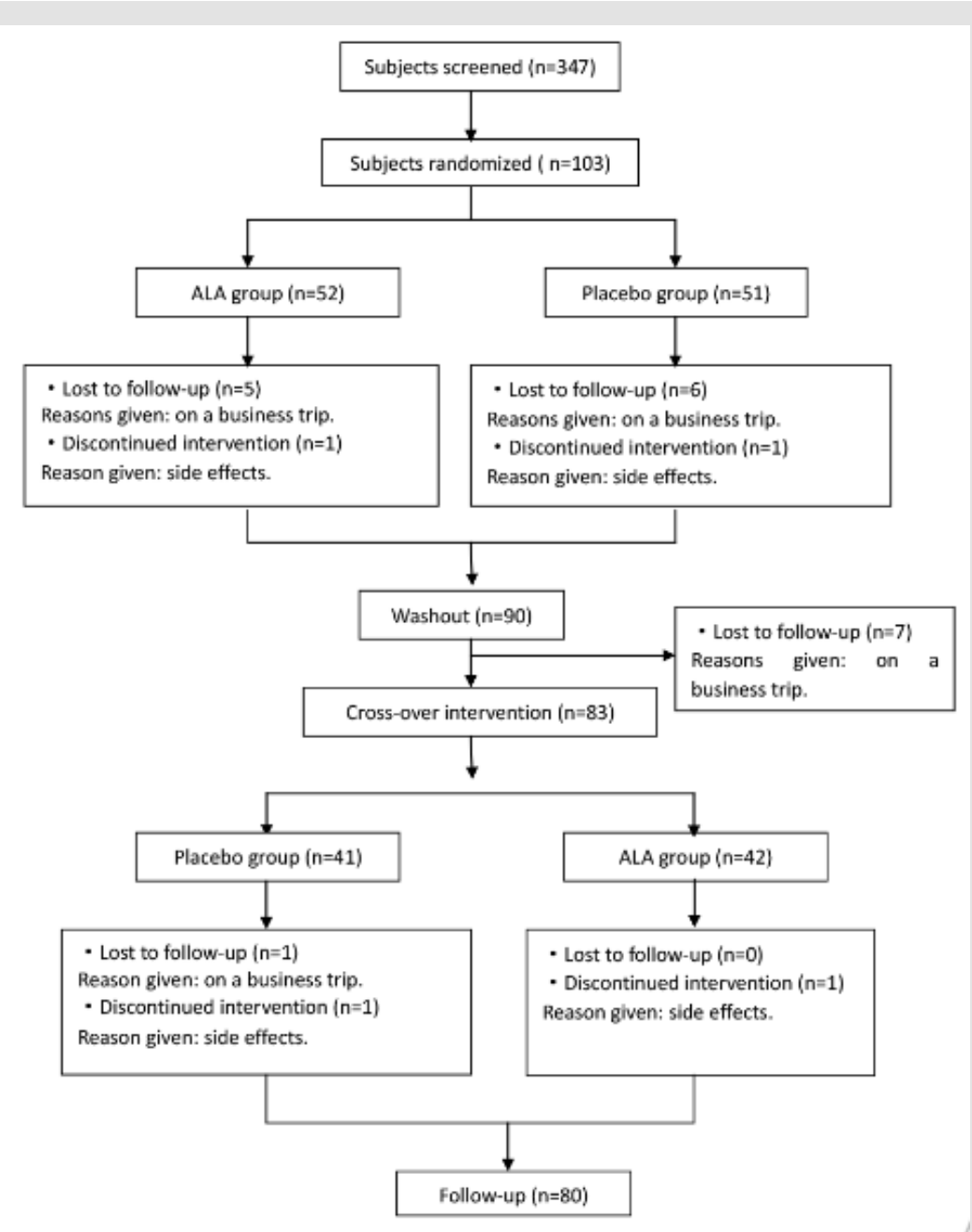

Figure 1: Participant flow chart.

Note: ALA: a-lipoic acid.

\section{Assessment}

Before enrollment, all subjects underwent a medical examination. Baseline characteristics were collected after signed informed consent was obtained, and data were also collected the end of the 8-, 12- (washout period), and 20-week interventions. The baseline assessment included: general information (demographic characteristics, socio-economic data, and personal and family history); dietary analysis (24-hour recalls and food frequency); physical examination (height, body weight, and waist and hip circumference); blood pressure; fasting and 2-hour postmeal insulin levels; $75 \mathrm{~g}$ oral glucose tolerance test; and blood biochemical indices. The evaluation at the end of the 8-, 12- and 20 week interventions included: physical examination (height, body weight, and waist and hip circumference); blood pressure; pulse wave velocity (PWV); CRP, interleukin-6 (IL-6); and fasting plasma glucose (FPG) and insulin (FINS). All investigators were specially trained and had to pass the qualifying test before participating in the study. All indices were assessed using unified methods by the specified investigators.

\section{General Information}

General information included: age, sex, race, profession, economic conditions, education, and personal or family history including hypertension, CA(H)D, hyperlipidemia, obesity and stroke, family member's diseases and corresponding treatment, diet, and exercise. Dietary measurements were based on food frequency and 24-hour dietary recall methods. The food frequency was analyzed using a food frequency questionnaire according to the 2010 Chinese National Nutrition and Health Survey, including the frequency and amount of food and condiment consumption in the last three months of the study. The 24-hour dietary recall method investigated the diet consumed the previous day. For each subject, the daily intake of various foods, nutrients, and proportions of energy from sugar, protein, and fat were calculated from the food composition tables based on the primary data from the questionnaires. An investigation of medicine use was carried out at the end of the intervention period, and the remaining tablets were collected. Adverse effects (AEs) during the study were recorded by a questionnaire, and medication compliance was assessed based on the remaining tablets. 


\section{Physical Examination}

Physical examination included height, body weight, and waist and hip circumference which was assessed on an empty stomach, with shoes and heavy clothing removed. BMI was calculated as weight $(\mathrm{kg}) /$ height $\left(\mathrm{m}^{2}\right)$. Blood pressure was measured according to the recommendations of the American Heart Association, and right upper arm blood pressure was observed using a mercury sphygmomanometer by qualified investigators.

\section{Pulse Wave Velocity (PWV) Measurement}

The brachial-ankle pulse wave velocity (baPWV) was measured using a VP-1000 device (Model BP203RPE, type II PWV/ABI; Omron, Japan) by a specialist from Xinjiang Medical University, Urumqi, China.

\section{Blood Collection and Biochemical Indices Measurement}

Venous blood was collected in the early morning on an empty stomach for at least 8 hours. The reagents for blood glucose, TC, triglyceride (TG) and HDL-C determination, as well as calibration and quality controls, were supplied by Shanghai Fosun Long March Medical Science Co. Ltd, Shanghai, China, and these biochemical indices were measured on an TBA-120 automatic biochemical analyzer (Toshiba Medical Systems, Japan). Plasma insulin was detected using an automatic Access II chemiluminescence system (Beckman Coulter Inc., Fullerton, CA, USA), and the related reagents, calibration, and quality controls were supplied by the same company. All blood biochemical indices were determined on the same day of blood collection at the Medical Center of the People's Hospital of Kalamay, Xinjiang, China. Levels of CRP and IL-6 were quantified by radioimmunoassay (RIA) using a Sn-695B RIA counter (Shanghai Hesuo Rihuan Photoelectric Instrument Co., Ltd, Shanghai, China) at the RIA Technology Development Center of PLA General Hospital, Beijing, China, and the related RIA kits for radioiodinated (125I) leptin, CRP, and 125I IL-6 were manufactured by the same center.

\section{Statistical Analysis}

Databases were created in Microsoft Access and error correction was carried out after double data entry. All statistical analyses were performed using Stata version 11.0, and all measurement data were expressed as mean \pm standard deviation (SD). After normal transformation, the non-normal distribution data were conducted with statistical disposal. Baseline data were compared with independent sample t-tests. After adjustment of treatment sequence and period, baseline values, sex and age, mixed effects models were used to compare the efficacy between the two groups. p values $<0.05$ were considered statistically significant. Intention-to-treat was used for statistical analysis. For subjects who withdrew with measurements available before the intervention but not after the intervention, the differences were considered as ' 0 ' during statistical analysis. The mixed effects models were used to analyze the relationships between independent variables (baseline characteristics, age, sex, treatment sequence and period) and dependent variables (differences in the levels of baPWV, SBP, DBP, CRP, IL-6, and before and after intervention). The power analysis was made using the power and sample size program. After intervention for 8 weeks, if the difference in average values of each indicator between ALA and placebo groups was speculated to be 0.2 SD, a sample size of 103 subjects in each group would have a statistical power $>80 \%(\mathrm{p}<0.01)$.

\section{Result}

\section{Comparison of Baseline Characteristics}

Enrolled subjects were randomly divided into two groups in a 1:1 ratio balanced for gender. There were 52 subjects in the ALA group with a mean age of $42.12 \pm 7.59$ years, and 51 subjects in the placebo group with a mean age of $41.76 \pm 6.84$ years. Baseline characteristics, including physical examination, biochemical indices, and inflammation, were found to be comparable between the two groups (Tables 1).

Table 1: Comparison of the baseline information in the two groups (mean $\pm \mathrm{SD}$ ).

\begin{tabular}{|c|c|c|c|c|}
\hline Variable & ALA Group & Placebo Group & $T$ value $(x 2)$ & p Value \\
\hline Age (years) & $42.12 \pm 7.59$ & $41.76 \pm 6.84$ & 0.246 & 0.806 \\
\hline Male: Female & $32: 20: 00$ & $31: 20: 00$ & 0.006 & 0.937 \\
\hline $\mathrm{BMI}^{\mathrm{a}}\left(\mathrm{kg} / \mathrm{m}^{2}\right)$ & $28.53 \pm 2.53$ & $28.70 \pm 2.59$ & 0.355 & 0.723 \\
\hline Hip c $(\mathrm{cm})$ & $103.93 \pm 4.95$ & $104 \pm 4.67$ & -0.077 & 0.939 \\
\hline $\mathrm{Wc}(\mathrm{cm})$ & $95.05 \pm 8.72$ & $96.01 \pm 7.10$ & -0.607 & 0.546 \\
\hline $\mathrm{SBP}(\mathrm{mmHg})$ & $120.12 \pm 7.36$ & $118.55 \pm 6.18$ & 1.173 & 0.244 \\
\hline DBP (mmHg) & $82 \pm 6.13$ & $82.35 \pm 6.65$ & -0.275 & 0.784 \\
\hline $\mathrm{TC}(\mathrm{mmol} / \mathrm{l})$ & $5.18 \pm 0.79$ & $5.43 \pm 1.05$ & -1.377 & 0.172 \\
\hline HDL-C (mmol/l) & $1.31 \pm 0.30$ & $1.23 \pm 0.30$ & 1.338 & 0.184 \\
\hline $\mathrm{TG}^{\mathrm{b}}(\mathrm{mmol} / \mathrm{l})$ & $2.16 \pm 1.23$ & $2.24 \pm 1.45$ & -0.042 & 0.967 \\
\hline FBG (mmol/l) & $5.44 \pm 0.77$ & $5.31 \pm 0.79$ & 0.831 & 0.408 \\
\hline FINS $^{a}(\mu \mathrm{IU} / \mathrm{ml})$ & $9.07 \pm 6.39$ & $8.46 \pm 5.45$ & 0.401 & 0.689 \\
\hline $\mathrm{BaPWV}(\mathrm{cm} / \mathrm{s})$ & $1438.60 \pm 197.55$ & $1382.66 \pm 178.47$ & -1.595 & 0.114 \\
\hline
\end{tabular}




\begin{tabular}{|l|c|c|c|c|}
\hline IL-6 $(\mathrm{pg} / \mathrm{ml})$ & $129.60 \pm 33.49$ & $142.53 \pm 70.57$ & -1.179 & 0.241 \\
\hline CRP $(\mu \mathrm{g} / \mathrm{ml})$ & $7.41 \pm 7.42$ & $8.22 \pm 8.96$ & 0.504 & 0.616 \\
\hline
\end{tabular}

Note: BMI: Body Mass Index; Hip c: Hip Circumference; Wc: Waist and hip circumference; SBP: Systolic Blood Pressure; DBP: Diastolic Blood Pressure; TC: Total Cholesterol; HDL-C: High Density Lipoprotein Cholesterol; TG: Triglyceride; FBG: Fasting Blood Glucose; FINS: Fasting Insulin; BaPWV: Brachial-Ankle Pulse Wave Velocity; IL-6: Interleukin-6; CRP: C-Reactive Protein; a: Square Inverse Transformation; b: Logarithm Transformation

\section{Comparison of Baseline Dietary Intake}

Baseline dietary intake for energy and the three main nutrients, including fat, protein and carbohydrates, as well as antioxidant nutrients such as vitamins $\mathrm{A}, \mathrm{C}$, and $\mathrm{E}$ and selenium, zinc and manganese, were distributed evenly and comparable between the two groups (Table 2).

Table 2: Comparison of the baseline dietary intake in the two groups (mean $\pm \mathrm{SD}$ ).

\begin{tabular}{|c|c|c|c|}
\hline Variable & ALA group $(\mathbf{n = 5 2})$ & Placebo Group (n=51) & p Value \\
\hline Energy $^{\mathrm{a}}(\mathrm{Kcal})$ & $3096.85 \pm 1155.46$ & $3230.45 \pm 1175.19$ & 0.496 \\
\hline Protein $^{\mathrm{a}}(\mathrm{g})$ & $114.34 \pm 49.97$ & $129.76 \pm 62.51$ & 0.195 \\
\hline Fat $^{\mathrm{a}}(\mathrm{g})$ & $138.3 \pm 67.82$ & $137.07 \pm 71.93$ & 0.924 \\
\hline Carbohydrate $^{\mathrm{a}}(\mathrm{g})$ & $375.27 \pm 164.89$ & $392.27 \pm 173.48$ & 0.588 \\
\hline Vitamin A $^{\mathrm{a}}(\mu \mathrm{g})$ & $1681.17 \pm 1321.02$ & $1861.16 \pm 1414.45$ & 0.314 \\
\hline Vitamin C $^{\mathrm{a}}(\mathrm{mg})$ & $211.17 \pm 133.47$ & $237.78 \pm 127$ & 0.151 \\
\hline Vitamin $^{\mathrm{a}}(\mathrm{mg})$ & $76.33 \pm 55.48$ & $69.12 \pm 45.41$ & 0.758 \\
\hline Zinc $^{\mathrm{a}}(\mathrm{mg})$ & $20.76 \pm 9.98$ & $28.01 \pm 32.21$ & 0.165 \\
\hline Selenium $^{\mathrm{a}}(\mu \mathrm{g})$ & $53.48 \pm 26.67$ & $56.79 \pm 26.32$ & 0.4 \\
\hline Manganese $^{\mathrm{b}}(\mathrm{mg})$ & $19.17 \pm 21.92$ & $25.65 \pm 39.6$ & 0.849 \\
\hline
\end{tabular}

Note: a: logarithm transformation; b: inverse transformation of square root.

\section{Intervention Studies}

Compared with the baseline values, a reduction in the levels of IL-6, CRP, SBP and baPWV were observed after oral administration of ALA for 8 weeks in the ALA group, which were decreased by $22.51 \pm 37.1 \mathrm{pg} / \mathrm{ml}, 0.85 \pm 5.92 \mu \mathrm{g} / \mathrm{ml}, 1.25 \pm 8.95 \mathrm{mmHg}$, $26.30 \pm 117.23 \mathrm{~cm} / \mathrm{s}$, respectively. While DPB levels in the ALA group increased by $5.44 \pm 8.96 \mathrm{mmHg}$, in comparison with those at baseline. After 8 weeks of treatment, the levels of DBP, SPB, baPWV in the placebo group increased respectively, by $2.29 \pm 9.142 \mathrm{mmHg}$, $7.19 \pm 10.08 \mathrm{~mm} \mathrm{Hg}, 4.23 \pm 120.78 \mathrm{~cm} / \mathrm{s}$, compared to baseline values, whereas levels of IL- 6 and CRP decreased by $30.39 \pm 53.47 \mathrm{pg} / \mathrm{ml}$ and $0.83 \pm 5.96 \mu \mathrm{g} / \mathrm{ml}$, respectively. Based on mixed-effects models, there were significant changes in the levels of baPWV, SBP, DBP, CRP, IL- 6 between the two groups before and after the intervention $(\mathrm{p}<0.05)$. After adjusting for differences in sex, age, treatment sequence and period, the results of mixed-effects models showed that the differences of SBP in the two groups were significant before and after the intervention ( $\mathrm{p}<0.05)$, whereas there were no obvious differences for changes in the levels of baPWV, DBP, CRP and IL-6 between the two groups (Table 3), ( $p>0.05)$.

Table 3: Intent-to-treat analysis of the markers of inflammation and blood pressure by adjusted mixed-effects models in the two groups (mean $\pm \mathrm{SD})$.

\begin{tabular}{|c|c|c|c|c|c|c|c|c|c|}
\hline \multirow{2}{*}{ Variable } & \multirow{2}{*}{$\begin{array}{l}\text { ALA Group } \\
(n=103)\end{array}$} & \multirow{2}{*}{$\begin{array}{l}\text { Placebo Group } \\
\quad(n=103)\end{array}$} & \multicolumn{7}{|c|}{ p Value } \\
\hline & & & Baseline & Age & Sex & Treatment & Sequence & Period & Model \\
\hline IL-6 (pg/ml) & $-22.51 \pm 37.11$ & $-30.39 \pm 53.47$ & $<0.001$ & 0.891 & 0.973 & 0.552 & 0.794 & $<0.001$ & $<0.001$ \\
\hline$\triangle \mathrm{CRP}(\mu \mathrm{g} / \mathrm{ml})$ & $-0.85 \pm 5.92$ & $-0.83 \pm 5.96$ & $<0.001$ & 0.224 & 0.012 & 0.884 & 0.621 & 0.785 & $<0.001$ \\
\hline$\triangle \mathrm{SBP}(\mathrm{mmHg})$ & $-1.25 \pm 8.59$ & $2.29 \pm 9.12$ & 0 & 0.688 & 0.272 & 0.029 & 0.078 & 0.358 & 0 \\
\hline$\triangle \mathrm{DBP}(\mathrm{mmHg})$ & $5.44 \pm 8.96$ & $7.19 \pm 10.08$ & 0 & 0.129 & 0.1 & 0.121 & 0.187 & 0 & 0 \\
\hline$\triangle \mathrm{baPWV}(\mathrm{cm} / \mathrm{s})$ & $-26.30 \pm 117.23$ & $4.23 \pm 120.78$ & $<0.001$ & 0.122 & 0.023 & 0.111 & 0.744 & 0.89 & $<0.001$ \\
\hline
\end{tabular}

Note: $\triangle$ : differences of levels after and before the intervention; a: logarithm transformation; ALA: a-lipoic acid.

\section{Discussion}

Obesity has been considered as a state of chronic OS and inflammation. OS and inflammatory responses in obese subjects may provide a common mechanism for the pathogenesis of obesity-induced CVD. IL-6 is a multifunctional cytokine and has been reported to be involved in regulating immune inflammatory responses and glucolipid metabolism. As an important mediator of immune responses, IL- 6 has a central role in the regulation 
of inflammatory responses, which has effects on vascular walls leading to vascular wall injury. In addition, IL-6 is considered to have a crucial role in insulin resistance and atherosclerosis. CRP is an acute phase protein produced by the liver and can react with C-polysaccharide isolated from pneumococcal cell walls [22]. Several studies have suggested that CRP is a strong independent predictor of future cardiovascular events $[23,24]$. As a hepatocytestimulating factor, CRP is a sensitive marker of inflammatory responses [25] and has been widely used to reflect inflammatory responses in clinical practice $[26,27]$.

A recent study showed that $O S$ is the common pathway involving multiple risk factors for atherosclerosis, and OS-induced vascular inflammation served as an early indicator of atherosclerosis. OS can activate nuclear transcription factor $\kappa \beta$ (NF- $\kappa \beta)$ which can induce the expression of inflammatory cytokine genes leading to vascular inflammation, which suggests that NF- $\kappa \beta$ has a central role in the regulation of immunity and inflammatory responses [16,17]. ALA is a disulphide compound found in mitochondria as the coenzyme for the dehydrogenase complex, a coenzyme important in glucose oxidative metabolism. Similar to vitamins $\mathrm{C}$ and $\mathrm{E}, \mathrm{ALA}$ is a powerful antioxidant and free-radical scavenger that can bind heavy metals and regenerate other antioxidants. In addition, ALA can inhibit NF$\kappa \beta$ in vascular inflammatory responses, protecting the function of the vascular endothelium and preventing atherosclerosis [28].

A previous study has shown that ALA can inhibit the expression of intercellular adhesion molecule 1 and vascular cell adhesion molecule 1, preventing vascular endothelial injury caused by OS and protecting endothelial function [29]. Sola $S$ et al. evaluated the ability of ALA administered at a daily dose of $300 \mathrm{mg}$ in patients with MetS, and found that compared with the placebo group, ALA significantly reduced levels of IL- 6 and improved endothelial function; however, no significant differences were found in the reduction of 8-iso-PGF2 $\alpha$ levels [19]. In the present study, after oral administration of ALA at a daily dose of $1200 \mathrm{mg}$ for 8 weeks, no significant reduction in markers of inflammation were observed in comparison with those in the placebo group. There are a number of possible explanations for the results. First, the antioxidant effects of ALA have mainly been demonstrated in in vitro studies. Although strong in vitro evidence supports the role of ALA as a powerful antioxidant, it remains debatable whether ALA can scavenge free radicals effectively in vivo.

Because ALA transiently accumulates in vivo and is rapidly catabolized. In cell culture studies, the intracellular concentration of ALA following an oral dose was found to be several times higher than that in the plasma or tissues. So, typical cell culture conditions likely overestimate the direct antioxidant capacity of ALA. Alternatively, ALA may act indirectly to maintain antioxidant status by enhancing the synthesis of endogenous antioxidants, rather than through a direct-acting antioxidant role in the body [30]. Glutathione (GSH) is a natural thiol antioxidant and substrate for detoxification enzymes, and studies have shown that ALA treatment can enhance
GSH levels [31,32]. Secondly, oral administration of ALA has been shown to be less effective than intravenous injection. Compared with oral administration, intravenous infusion can increase insulin sensitivity in patients with T2D in a dose-dependent manner [33]. Finally, in the present study, the antioxidant properties of oral ALA intake at a daily dose of $1200 \mathrm{mg}$ were not strong enough to inhibit NF- $\kappa \beta$ which could lead to vascular inflammation, so the pathways involved in the inflammatory responses were still active and the levels of inflammatory markers were not significantly reduced.

Recently, attention has focused on arterial elasticity in the development of hypertension. The endothelial cells covering the inner surface of vascular walls can produce, activate, and release various vasoactive substances, such as nitric oxide (NO), prostacyclin, endothelin, and endothelium-dependent vascular contraction factors, which can regulate cardiovascular function. OS can increase the number of oxyradicals and speed up the inactivation of NO, affecting arterial elasticity and structure. Reduced arterial elasticity and increased PWV can lead to an earlier reflection of the pressure back to the central arteries, earlier in the systolic phase than in the diastolic phase, causing a delayed pressure wave in the diastolic phase, which can increase the pulse pressure due to elevated DBP and decreased SBP. The elasticity of vascular walls is regulated by NO synthesized by endothelial NO synthase (eNOS). So, the inactivation of eNOS could lead to endothelial dysfunction due to impaired NO production, which is characterized by reduced vasodilatation and a pro-inflammatory and pro-thrombotic phenotype, as well as OS-induced endothelial dysfunction. In this case, antioxidants like vitamin C and ALA can improve the redox status in plasma and endothelium-dependent vasodilatation regulated by NO. This is because ALA can activate the AT3K/Akt signaling pathway, resulting in the activation of eNOS.

Endothelin-1 is a vasoconstrictor produced by endothelial cells, and it has been shown that ALA can inhibit endothelin-1 overproduction in the kidney and blood vessels. Recent studies have also shown that ALA can improve NO synthesis, i.e., the main endothelial-derived vasodilator. Moreover, the onset of essential hypertension is also associated with abnormal ion transport across cell membranes. Various specific ion channels that are found in vascular smooth muscle cells maintain the dynamic balance of $\mathrm{K}^{+}, \mathrm{Na}^{+}$, and $\mathrm{Ca}^{2+}$ ions between the intracellular and extracellular environments. The abnormality in ion transport increases the concentrations of intracellular $\mathrm{Na}^{+}$and $\mathrm{Ca}^{2+}$, reduces membrane potential, and activates the process of excitation-contraction coupling in smooth muscle cells (SMCs), which then increase vasoconstrictor reactivity and vascular resistance accompanied by an increase in SMC size. In animal models, ALA treatment given to rats with hypertension can reduce the release of cytoplasmic calcium and slow the changes in renal vessels, enabling DBP to return to normal levels. In the clinic, ALA in combination with acetylL-carnitine has been used in the treatment of MetS complicated by hypertension to enhance the anti-hypertensive effects associated 
with reduced DBP. By contrast, sola et al. found no significant changes in blood pressure compared after oral administration of ALA at a daily dose of $300 \mathrm{mg}$ in patients with MetS, compared with the placebo group ( $p>0.005)$ [19].

In the present study, overweight or obese subjects who completed 8 weeks of treatment with ALA showed a reduction in DBP. This is probably because ALA can activate eNOS, improve endothelial function, promote the release of NO, inhibit excessive endothelin-1 produced by the kidney and blood vessels, resulting in vasodilatation. Moreover, the possible mechanisms are also associated with increased levels of endogenous antioxidant markers caused by ALA treatment, which can maintain the dynamic balance of $\mathrm{K}^{+}, \mathrm{Na}^{+}$, and $\mathrm{Ca}^{2+}$ on both sides of the cell membrane, correcting the abnormal ion transport. Epidemiological and other related studies have suggested that increased arterial stiffness is an independent risk factor for CVD and is also positively associated with atherosclerosis [34]. PWV is a useful measure of arterial stiffness and has been shown to be of great value in the evaluation of CVD [35,36]; therefore, we used PWV as one of the arterial stiffness indices in our clinic. In the present study, after treatment with ALA for 8 weeks, PWV in the ALA group was reduced to some extent compared with the placebo group, but the difference between the two groups was not significant ( $p>0.05)$. This might be due to the weaker antioxidant activity of ALA which could not completely inhibit NF- $\kappa \beta$ so that the pathways involved in the inflammatory responses were still active and atherosclerosis had not been fully reversed. Above all, our results suggested that overweight or obese subjects who received ALA at a daily dose of $1200 \mathrm{mg}$ for 8 weeks showed a reduction in body weight. Nevertheless, further studies are needed to determine the possible mechanisms.

There were some limitations in the current study. The sample size may not be large enough. The SD of each measurement data was large, whereas differences in the means between the two groups were lower. Large intra-group differences and lower intergroup differences showed that the estimation of sample size in our study was lower than assumed. In addition, the length of the trial was relatively short. Another clinic trial has determined the effects of $600 \mathrm{mg}$ of ALA given intravenously over 12 weeks [21]. Although we adopted a double-dose regime in the present study, orally supplied ALA for a shorter time (8 weeks) may be insufficient to exert its effects.

\section{Conclusion}

At present, only a limited number of studies have examined the effects of antioxidants on cardiovascular risk factors in obese human subjects exist. In the present study, we carried out a randomized, double-blind, placebo-controlled crossover clinical trial to determine whether oral intake of ALA could reduce blood pressure and inflammatory factors. We found that after treatment with oral ALA at a dose of $1200 \mathrm{mg}$ for 8 weeks, The SBP was reduced, which could be considered as a novel therapy for hypertension. Our study could also provide further guidance for future clinical trials, although the changes in the biomarkers of inflammation and PWV levels were not significant. In order to determine the stronger effects of ALA on cardiovascular risk factors, multicenter research with a larger sample size, longer time of treatment, or increased dose of ALA is required.

\section{Conflicts of Interest and Source of Funding}

All the author declare they have no confict of interests. This study was supported by the Science and Technology Bureau Xinjiang Karamay (SK2009-09). We thank all the doctors, nurses of People's Hospital of Kelamay city, the postgraduates of college of public health of xinjiang medical university and the volunteers who joined in this study. We also thank the MRI (US) company for the free ALA pill and placebo.

\section{References}

1. Kearney PM, Whelton M, Reynolds K, Muntner P, Whelton PK, et al. (2005) Global burden of hypertension: analysis of worldwide data. Lancet 365: 217-223.

2. Poirier P, Giles TD, Bray GA, Hong Y, Stern JS, et al. (2006) Obesity and cardiovascular disease: pathophysiology, evaluation, and effect of weight loss: an update of the 1997 American Heart Association Scientific Statement on Obesity and Heart Disease from the Obesity Committee of the Council on Nutrition, Physical Activity, and Metabolism. Circulation 113: 898-918.

3. Wild S, Roglic G, Green A, Sicree R, King H (2004) Global prevalence of diabetes: estimates for the year 2000 and projections for 2030. Diabetes Care 27: 1047-1053.

4. Kannel WB, Wilson PW, Nam BH, D’Agostino RB (2002) Risk stratification of obesity as a coronary risk factor. Am J Cardiol 90: 697-701.

5. Eckel RH, Barouch WW, Ershow AG (2002) Report of the National Heart Lung, and Blood Institute-National Institute of Diabetes and Digestive and Kidney Diseases Working Group on the pathophysiology of obesityassociated cardiovascular disease. Circulation 105: 2923-2928.

6. Keaney JF, Larson MG, Vasan RS, Wilson PW, Lipinska I, et al. (2003) Obesity and systemic oxidative stress: clinical correlates of oxidative stress in the Framingham Study. Arterioscler Thromb Vasc Biol 23: 434439.

7. Ceriello A, Motz E (2004) Is oxidative stress the pathogenic mechanism underlying insulin resistance, diabetes, and cardiovascular disease? The common soil hypothesis revisited. Arterioscler Thromb Vasc Biol 24: 816-823.

8. Vincent HK, Taylor AG (2006) Biomarkers and potential mechanisms of obesity-induced oxidant stress in humans. Int J Obes (Lond) 30: 400418.

9. Block G, Dietrich M, Norkus EP, Morrow JD, Hudes M, et al. (2002) Factors associated with oxidative stress in human populations. Am J Epidemiol 156: 274-285

10. Davi G, Guagnano MT, Ciabattoni G, Basili S, Falco A, et al. (2002) Platelet activation in obese women: role of inflammation and oxidant stress. JAMA 288: 2008-2014.

11. Zalba G, San Jose G, Moreno MU, Fortuno MA, Fortuno A, et al. (2001) Oxidative stress in arterial hypertension: role of $\mathrm{NAD}(\mathrm{P}) \mathrm{H}$ oxidase. Hypertension 38: 1395-1399.

12. Puddu P, Puddu GM, Cravero E, Rosati M, Muscari A (2008) The molecular sources of reactive oxygen species in hypertension. Blood Press 17: 7077. 
13. Visser M, Bouter LM, Mc Quillan GM, Wener MH, Harris TB (1999) Elevated C-reactive protein levels in overweight and obese adults. JAMA 282: 2131-2135.

14. Festa A, D’Agostino R, Williams K, Karter AJ, Mayer-Davis EJ, et al. (2001) The relation of body fat mass and distribution to markers of chronic inflammation. Int J Obes Relat Metab Disord 25: 1407-1415.

15. Mohamed-Ali V, Goodrick S, Rawesh A, Katz DR, Miles JM, et al. (1997) Subcutaneous adipose tissue releases interleukin-6, but not tumor necrosis factor-alpha, in vivo. J Clin Endocrinol Metab 82: 4196-4200.

16. Ross R (1999) Atherosclerosis--an inflammatory disease. N Engl J Med 340: 115-126.

17. Hansson GK (2005) Inflammation, atherosclerosis, and coronary artery disease. N Engl J Med 352: 1685-1695.

18. De Ferranti S, Rifai N (2002) C-reactive protein and cardiovascular disease: a review of risk prediction and interventions. Clin Chim Acta 317: 1-15.

19. Sola S, Mir MQ, Cheema FA, Khan-Merchant N, Menon RG, et al. (2005) Irbesartan and lipoic acid improve endothelial function and reduce markers of inflammation in the metabolic syndrome: results of the Irbesartan and Lipoic Acid in Endothelial Dysfunction (ISLAND) study. Circulation 111: 343-348.

20. Ziegler D, Reljanovic M, Mehnert H, Gries FA (1999) Alpha-lipoic acid in the treatment of diabetic polyneuropathy in Germany: current evidence from clinical trials. Exp Clin Endocrinol Diabetes 107: 421-430.

21. Ziegler D, Nowak H, Kempler P, Vargha P, Low PA (2004) Treatment of symptomatic diabetic polyneuropathy with the antioxidant alpha-lipoic acid: a meta-analysis. Diabet Med 21: 114-121.

22. Pannitteri G, Marino B, Campa PP, Martucci R, Testa U, et al. (1997) Interleukins 6 and 8 as mediators of acute phase response in acute myocardial infarction. Am J Cardiol 80: 622-625.

23. Ridker PM, Rifai N, Rose L, Buring JE, Cook NR (2002) Comparison of C-reactive protein and low-density lipoprotein cholesterol levels in the prediction of first cardiovascular events. N Engl J Med 347: 1557-1565.

24. Ridker PM (2001) High-sensitivity C-reactive protein: potential adjunct for global risk assessment in the primary prevention of cardiovascular disease. Circulation 103: 1813-1818.

ISSN: 2574-1241

DOI: $10.26717 /$ BJSTR.2020.28.004591

Meiyu Zhang. Biomed J Sci \& Tech Res

(C) This work is licensed under Creative

Submission Link: https://biomedres.us/submit-manuscript.php
25. Patel VB, Robbins MA, Topol EJ (2001) C-reactive protein: a 'golden marker' for inflammation and coronary artery disease. Cleve Clin J Med 68: 521-524, 527-534.

26. Dietrich M, Block G, Hudes M, Morrow JD, Norkus EP, et al. (2002) Antioxidant supplementation decreases lipid peroxidation biomarker $\mathrm{F}(2)$-isoprostanes in plasma of smokers. Cancer Epidemiol Biomarkers Prev 11: 7-13.

27. Esposito K, Pontillo A, Di Palo C, Giugliano G, Masella M, et al. (2003) Effect of weight loss and lifestyle changes on vascular inflammatory markers in obese women: a randomized trial. JAMA 289: 1799-1804.

28. Packer L, Witt EH, Tritschler HJ (1995) alpha-Lipoic acid as a biological antioxidant. Free Radic Biol Med 19: 227-250.

29. Chaudhary P, Marracci GH, Bourdette DN (2006) Lipoic acid inhibits expression of ICAM-1 and VCAM-1 by CNS endothelial cells and T cell migration into the spinal cord in experimental autoimmune encephalomyelitis. J Neuroimmunol 175: 87-96.

30. Shay KP, Moreau RF, Smith EJ, Smith AR, Hagen TM (2009) Alpha-lipoic acid as a dietary supplement: molecular mechanisms and therapeutic potential. Biochim Biophys Acta 1790: 1149-1160.

31. Bast A, Haenen GR (1988) Interplay between lipoic acid and glutathione in the protection against microsomal lipid peroxidation. Biochim Biophys Acta 963: 558-561.

32. Busse E, Zimmer G, Schopohl B, Kornhuber B (1992) Influence of alpha-lipoic acid on intracellular glutathione in vitro and in vivo. Arzneimittelforschung 42: 829-831.

33. Evans JL, Goldfine ID (2000) Alpha-lipoic acid: a multifunctional antioxidant that improves insulin sensitivity in patients with type 2 diabetes. Diabetes Technol Ther 2: 401-413.

34. Farrar DJ, Bond MG, Riley WA, Sawyer JK (1991) Anatomic correlates of aortic pulse wave velocity and carotid artery elasticity during atherosclerosis progression and regression in monkeys. Circulation 83:1754-1763.

35. Shimizu H (2005) Brachial-ankle pulse wave velocity. Intern Med 44: 688-689.

36. Yamashina A, Tomiyama H, Takeda K, Tsuda H, Arai T, et al. (2002) Validity, reproducibility, and clinical significance of noninvasive brachialankle pulse wave velocity measurement. Hypertens Res 25: 359-364.

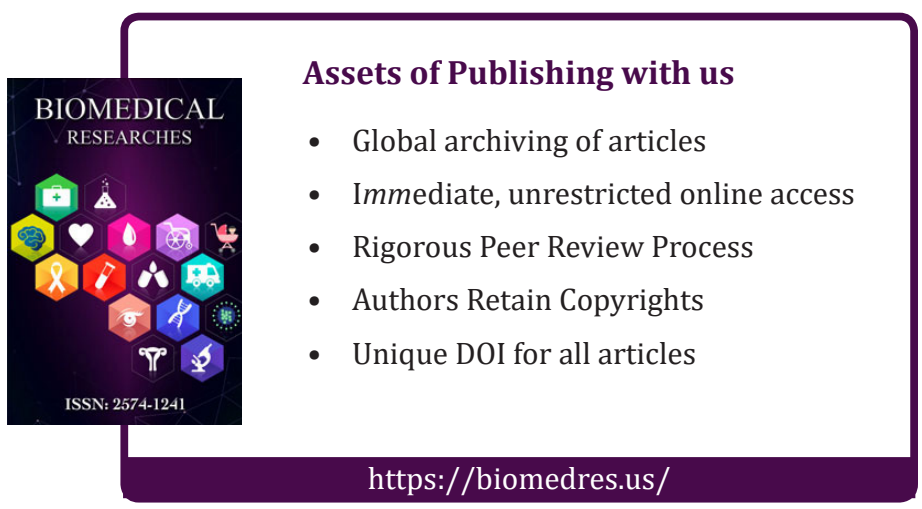

